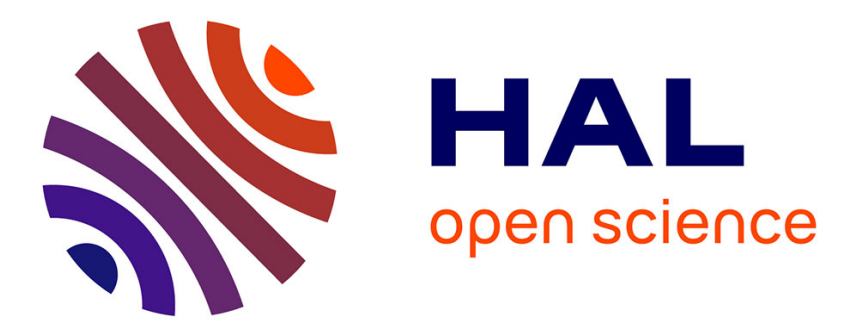

\title{
Bayesian inference for the ultrasonic characterization of rigid porous materials using reflected waves by the first interface
}

\author{
R. Roncen, Z.E. A Fellah, F. Simon, E. Piot, M. Fellah, Erick Ogam, C. \\ Depollier
}

\section{To cite this version:}

R. Roncen, Z.E. A Fellah, F. Simon, E. Piot, M. Fellah, et al.. Bayesian inference for the ultrasonic characterization of rigid porous materials using reflected waves by the first interface. Journal of the Acoustical Society of America, 2018, 144 (1), pp.210-221. 10.1121/1.5044423 . hal-01836022

\section{HAL Id: hal-01836022 \\ https://hal.science/hal-01836022}

Submitted on 25 Jul 2018

HAL is a multi-disciplinary open access archive for the deposit and dissemination of scientific research documents, whether they are published or not. The documents may come from teaching and research institutions in France or abroad, or from public or private research centers.
L'archive ouverte pluridisciplinaire HAL, est destinée au dépôt et à la diffusion de documents scientifiques de niveau recherche, publiés ou non, émanant des établissements d'enseignement et de recherche français ou étrangers, des laboratoires publics ou privés. 


\title{
Bayesian Inference for the Ultrasonic Characterization of Rigid Porous Materials Using Reflected Waves by the First Interface
}

\author{
R. Roncen*1 ${ }^{* 1}$ Z.E.A. Fellah ${ }^{2}$, F. Simon ${ }^{1}$, E. Piot ${ }^{1}$, M. Fellah ${ }^{3}$, E. Ogam ${ }^{2}$, and C. Depollier ${ }^{\dagger 4}$ \\ ${ }^{1}$ ONERA / DMPE, Université de Toulouse, F-31055, Toulouse, France \\ ${ }^{2}$ LMA, CNRS, UMR 7031, Aix-Marseille Univ, Centrale Marseille, F-13402 Marseille Cedex 20, France \\ ${ }^{3}$ Laboratoire de Physique Théorique, Faculté de Physique, USTHB, BP 32 El Alia, Bab Ezzouar 16111, \\ Algeria \\ ${ }^{4}$ LUNAM Universite du Maine, UMR CNRS 6613 Laboratoire d'Acoustique de l'Universite du Maine UFR \\ STS Avenue O. Messiaen 72085 Le Mans CEDEX 09, France
}

\begin{abstract}
The purpose of this paper is to present a method for the ultrasonic characterization of airsaturated porous media, by solving the inverse problem using only the reflected waves from the first interface to infer the porosity, the tortuosity and the viscous and thermal characteristic lengths. The solution of the inverse problem relies on the use of different reflected pressure signals obtained under multiple obliquely incident waves, in the time domain. In this paper, the authors propose to solve the inverse problem numerically with a first level Bayesian inference method, summarizing the knowledge on the inferred parameters in the form of posterior probability densities, exploring these densities using a Markov-Chain Monte-Carlo approach. Despite their low sensitivity to the reflection coefficient, it is still possible to extract the knowledge of the viscous and thermal characteristic lengths, allowing the simultaneous determination of all the physical parameters involved in the expression of the reflection operator. To further constrain the problem and guide the inference, the knowledge of a particular incident angle is used at one's advantage in order to more precisely define the thermal length, by effectively yielding a statistical relationship between tortuosity and characteristic lengths ratio.
\end{abstract}

\section{Introduction}

Porous materials find widespread applications, ranging from hydrology [1] to medicine (cancellous bone [2]) and sound absorption [3]. The estimation of their micro-structural properties, such as porosity, tortuosity and characteristic lengths, plays a crucial role in the characterization of their physical behavior. One common goal of these areas of physics is the non intrusive, robust and cheap inference of all the required intrinsic properties of porous media that are necessary to describe their physical behavior. Due to the inherent measurement system noise, the quantitative assessment of the uncertainty of the identified parameters is equally important.

The most widespread model to characterize wave propagation inside poroelastic media is Biot's model [4, 5, 6, 7], which accounts for the presence of three different waves inside the poroelastic material: two compressional waves and one shear wave. In some applications, one is mostly interested in acoustic propagation in rigid porous media where the solid phase is immobile and only the airborne compressional wave propagates; this configuration is the focus of the present study. The equivalent fluid model of Johnson-Champoux-Allard (JCA [8, 9]) is widely used for sound propagation in rigid porous media and requires the knowledge of 5 intrinsic material properties (porosity,

\footnotetext{
*remi.roncen@onera.fr

${ }^{\dagger}$ Permanent adress: LUNAM Universite du Maine, UMR CNRS 6613 Laboratoire d'Acoustique de l'Universite du Maine UFR STS Avenue O. Messiaen 72085 Le Mans CEDEX 09, France
} 
tortuosity, static viscous permeability, viscous and thermal characteristic lengths). The JCA model is well adapted to describe the acoustical behavior of porous materials with rigid skeletons having arbitrary pore shapes. The model is obtained by the connection between asymptotic developments in the low and high frequency regions. In the high frequency regime, where the viscous boundary layer is much smaller than the pore radius, the porosity, tortuosity and characteristic lengths are sufficient to define the porous wave behavior. These are the parameters inferred in the present study.

Different inverse problems for parameter identifications have been attempted in the past regarding air-saturated porous media, mostly in the audio frequency regime [10, 11, 12, 13, 14]. The largest unaddressed issues of these studies are the modeling deficiency at these frequencies and the scarcity of uncertainty considerations. Being in the high frequency domain allows the use of models that are exactly defined in the asymptotic limit, overcoming the modeling limitations of audiofrequency based inverse methods. In earlier studies, Fellah et al. [15, 16] formulated the reflection coefficient at the first interface of a rigid porous material in the high frequency limit. Multiple obliquely incident waves in the high frequency domain were used to obtain a direct relationship between the reflected waves and two parameters of interest, namely, the porosity and tortuosity. To extend the identification to other parameters, Fellah et al. [17] then introduced an ultrasonic characterization technique to identify the porosity, tortuosity and the two characteristic lengths, by using both reflected and transmitted waves and an inverse method based on time-domain signals, and by assuming a given ratio between the viscous and thermal characteristic lengths. Groby et al. [18] then developed an analytical method using both the reflected and transmitted signals in the ultrasonic regime to extract the values of the porosity, tortuosity and the viscous and thermal lengths, in the frequency domain; it was argued that for highly resistive materials, the method might yield wrong results. Later, Fellah et al. [19] focused on low resistivity materials and solved the inverse scattering problem in the ultrasonic regime for the transmission mode only, to identify the porosity, tortuosity and characteristic lengths using time domain signals. Using reflected waves from the first interface is particularly suited to characterizing highly resistive materials, because they have large reflection coefficients. However, the previous studies have not been able to yield the identification of the characteristic lengths using only reflected waves from the first interface. Only the porosity and tortuosity have been measured in this configuration [15, 16].

The primary aim of the present study is to extend the previous results of [15, 16] to identify the characteristic lengths of porous materials, using only the knowledge of obliquely reflected waves at the first material interface, in the high-frequency domain. In previous work [15, 16], the dispersive contribution to the reflection operator has been neglected. In this article, we take into account the dispersive part of the reflection coefficient (fractional derivative of order $-1 / 2$ in the time domain and $1 / \sqrt{j \omega}$ term in the frequency domain). The influence of the characteristic lengths on the reflection operator is taken into account, which in turn makes their identification possible when using only reflected waves.

The secondary objective is to apply to the ultrasonic time-domain identification process a statistical Bayesian inference method. This enables the systematical encompassing of measurement uncertainties and prior information into the identification of porous media properties, as was done previously in [20, 21] in the frequency domain at audio-frequencies when using impedance tube measurements as observational inputs.

The present article is organized as follow: the acoustical model is presented in Section II, where the high frequency limit of the JCA model is detailed and where the reflection operator formulation is given. The reflected wave is then expressed in the time domain. A critical incident angle is presented, relating the tortuosity to the characteristic length ratio. The inference methodology is described in Section III, where Bayes' theorem is recalled and the numerical exploration of the parameter space is detailed. Results for the inference of three widely different porous materials are presented in Section IV. Concluding remarks are presented in Section V. 


\section{$2 \quad$ Acoustical Model}

In the study of porous media, two different cases are typically considered. When the fluid and solid phases have coupled contribution to the acoustic attenuation in the material, the Biot model is used to describe the wave propagation in both the solid and fluid phases, resulting in the occurrence of two compressional and one rotational waves. When the solid phase can be assumed rigid, which is often the case in air-saturated porous media, and the frame motion can be neglected, one usually resorts to using an equivalent fluid model where only the compressional wave propagation in the fluid phase of the porous material is considered. The classical properties of density and compressibility are replaced by equivalent properties in order to take into account viscous and thermal dissipation in the pores. This is the approach we adopt in this work.

Let a macroscopically homogeneous rigid porous material of porosity $\phi$ be filled with a fluid of density $\rho_{f}$ and dynamic viscosity $\eta$. Assuming weak fluid-frame coupling and a wavelength much larger than the pore size, one can apply continuum mechanics to the fluid phase in the material and identify the whole porous medium as an equivalent fluid material of effective properties to account for viscous and thermal dissipation arising from the fluid-frame interaction. As initially proposed by Zwikker and Kosten [22], one can decouple the contribution of viscous and thermal dissipation in the pores. To do so, one defines the dynamic tortuosity $\tilde{\alpha}(t)$ that encompasses the viscous-inertial fluid/structure interactions, and the dynamic compressibility $\tilde{\beta}(t)$ that takes into account all the thermal fluid/structure interactions. In the time domain, these factors are operators and the equations controlling the wave behavior are the Euler equation and a constitutive equation, given in the time domain by

$$
\begin{array}{r}
\rho_{f} \int_{0}^{t} \tilde{\alpha}(t-\tau) \frac{\partial \boldsymbol{v}(\tau)}{\partial \tau} \mathrm{d} \tau=-\nabla p, \\
\frac{1}{K_{f}} \int_{0}^{t} \tilde{\beta}(t-\tau) \frac{\partial p(\tau)}{\partial \tau} \mathrm{d} \tau=-\nabla \cdot \boldsymbol{v} .
\end{array}
$$

In the previous equations, $\boldsymbol{v}$ is the macroscopic velocity and $p$ the macroscopic pressure, obtained by averaging the microscopic velocity and pressure fields over a representative elementary volume; $\rho_{f}$ is the fluid density and $K_{f}$ is the fluid bulk modulus.

Of primary interest to this study is to determine parameters defined in the high frequency limit, namely the porosity $\phi$, the tortuosity $\alpha_{\infty}$, the viscous characteristic length $\Lambda$ (unit $\mathrm{m}$, 8]) and the thermal characteristic length $\Lambda^{\prime}$ (unit $\mathrm{m},[9]$ ). The theoretical expressions of $\tilde{\alpha}(t)$ and $\tilde{\beta}(t)$ in the time domain, in the high frequency limit, are given by Johnson et al. [8] and Champoux \& Allard [9], and are written in time domain [23] as

$$
\begin{gathered}
\tilde{\alpha}(t)=\alpha_{\infty}\left(\delta(t)+\frac{2}{\Lambda} \sqrt{\frac{\eta}{\pi \rho_{f}}} \frac{H(t)}{\sqrt{t}}\right), \\
\tilde{\beta}(t)=\delta(t)+\frac{2(\gamma-1)}{\Lambda^{\prime}} \sqrt{\frac{\eta}{\pi \operatorname{Pr} \rho_{f}}} \frac{H(t)}{\sqrt{t}},
\end{gathered}
$$

where $\operatorname{Pr}$ is the Prandtl number, $\eta$ is the fluid dynamic viscosity, $\gamma=1.4$ is the fluid heat capacity ratio, $\delta(t)$ is the Dirac delta function (which represents the instantaneous, non-dispersive response of the material), and $H(t)$ is the Heaviside function $(H(t<0)=0, H(t \geq 0)=1)$, used for causality reasons. The time convolution of $t^{-1 / 2}$ is interpreted as a fractional derivative operator of order $\nu=1 / 2$, following the definition in Samko [24]:

$$
D^{\nu}[g(t)]=\frac{1}{\Gamma(-\nu)} \int_{0}^{t}(t-u)^{-\nu-1} g(u) \mathrm{d} u
$$

where $\Gamma(x)$ is the gamma function. These terms in $\tilde{\alpha}(t)$ and $\tilde{\beta}(t)$ represent the dispersive kernel, expressing the dispersive nature of the viscous and thermal effects in the pores at high frequencies. 


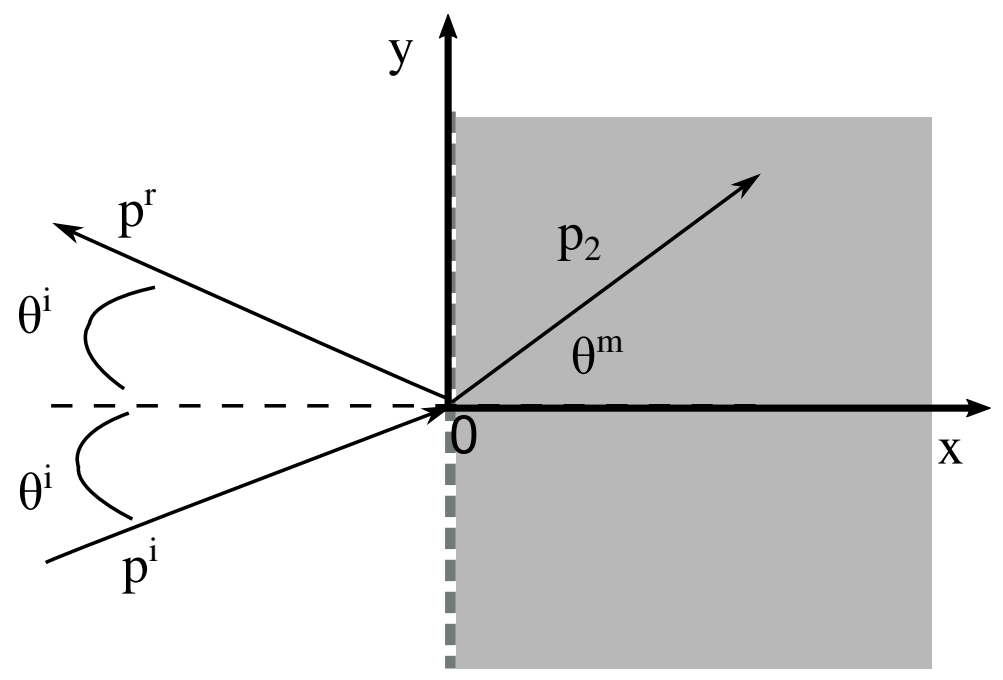

Figure 1: Schematic of the scattering problem

For a more in-depth analysis of fractional derivatives in acoustical modeling, the reader is referred to [25] and the references therein. Combining Eqs. 1] with Eqs. 2 yields

$$
\begin{gathered}
\rho_{f} \alpha_{\infty} \frac{\partial \boldsymbol{v}}{\partial t}+2 \frac{\rho_{f} \alpha_{\infty}}{\Lambda} \sqrt{\frac{\eta}{\pi \rho_{f}}} \int_{0}^{t} \frac{\partial \boldsymbol{v} / \partial \tau}{\sqrt{t-\tau}} \mathrm{d} \tau=-\nabla p, \\
\frac{1}{K_{f}} \frac{\partial p}{\partial t}+2 \frac{\gamma-1}{K_{f} \Lambda^{\prime}} \sqrt{\frac{\eta}{\pi \operatorname{Pr} \rho_{f}}} \int_{0}^{t} \frac{\partial p / \partial \tau}{\sqrt{t-\tau}} \mathrm{d} \tau=-\nabla \cdot \boldsymbol{v} .
\end{gathered}
$$

The dispersive nature of porous media is taken into account via a memory effect, meaning that part of the material's response is not instantaneous and is associated with a time delay.

\subsection{Reflection scattering operator}

The direct scattering problem requires finding the pressure field reflected by the first interface of the porous material. Let us consider here a rigid-frame, air-filled isotropic porous medium inside a semi-infinite region $0 \leq x<+\infty$, as depicted in Fig. 1. A sound pulse impinges on the medium from the left with an incident angle $\theta^{i}$. We are mostly interested here in determining the reflected wave, or, equivalently, the reflection scattering operator $\tilde{R}(t)$ that describes the relationship between incident $\left(p^{i}(\boldsymbol{x}, t)\right)$ and reflected $\left(p^{r}(\boldsymbol{x}, t)\right)$ plane waves, as

$$
p^{r}(\boldsymbol{x}, t)=\int_{0}^{t} \tilde{R}(\tau) p^{i}(\boldsymbol{x}, t-\tau) \mathrm{d} \tau .
$$

To derive the required operator, continuity of pressure and velocity on the first interface of the material is assumed, resulting in the interface conditions [26]

$$
p\left(0^{-}, t\right)=p\left(0^{+}, t\right), \quad \boldsymbol{v}\left(0^{-}, t\right)=\phi \boldsymbol{v}\left(0^{+}, t\right),
$$

where a porosity of 1 has been taken for the ambient fluid $(x<0)$, and where the - superscript stands for the left domain, and the + superscript stands for the right domain. In order to manipulate the following equations more easily, we make use of the Laplace transform method, assuming an idle medium as initial condition. We note $P(\boldsymbol{x}, s)($ resp. $V(\boldsymbol{x}, s))$ the Laplace transform of $p(\boldsymbol{x}, t)$ (resp. $v(\boldsymbol{x}, t)$ ) defined by

$$
P(\boldsymbol{x}, s)=\mathcal{L}[p(\boldsymbol{x}, t)]=\int_{0}^{\infty} \exp (-s t) p(\boldsymbol{x}, t) \mathrm{d} t
$$


and the inverse Laplace transform by $p(\boldsymbol{x}, t)=\mathcal{L}^{-1}[P(\boldsymbol{x}, s)]$. For the Laplace coefficients, we use the same symbols as the time operators, but remove the tilda. Using the relations $\mathcal{L}[\delta(t)]=1$ and $\mathcal{L}\left[\frac{H(t)}{\sqrt{\pi t}}\right]=\sqrt{\frac{1}{s}}$, one can transform the expressions of $\tilde{\alpha}(t)$ and $\tilde{\beta}(t)$ in the Laplace domain into

$$
\begin{array}{r}
\alpha(s)=\alpha_{\infty}\left(1+\frac{2}{\Lambda}\left(\frac{\eta}{s \rho_{f}}\right)^{1 / 2}\right) \\
\beta(s)=1+\frac{2(\gamma-1)}{\Lambda^{\prime}}\left(\frac{\eta}{s \operatorname{Pr} \rho_{f}}\right)^{1 / 2} .
\end{array}
$$

The expression of the pressure field in the region at the left of the material is the sum of the incident and reflected fields

$$
\begin{aligned}
p_{1}(\boldsymbol{x}, t)= & p^{i}\left(t-\frac{x}{c_{f}} \cos \theta^{i}-\frac{y}{c_{f}} \sin \theta^{i}\right) \\
& +p^{r}\left(t+\frac{x}{c_{f}} \cos \theta^{i}-\frac{y}{c_{f}} \sin \theta^{i}\right),
\end{aligned}
$$

where $c_{f}=\sqrt{K_{f} / \rho_{f}} \approx 340 \mathrm{~m} . \mathrm{s}^{-1}$ is the sound speed in air. In the Laplace domain, the fields on the left side of the material become, for $x<0$ [27]

$$
\left\{\begin{aligned}
P_{1}(\boldsymbol{x}, s)= & \left(e^{-s \frac{x}{c_{f}} \cos \theta^{i}}\right. \\
& \left.+R\left(s, \theta^{i}\right) e^{s \frac{x}{c_{f}} \cos \theta^{i}}\right) e^{-s \frac{y}{c_{f}} \sin \theta^{i}} \\
V_{1}(\boldsymbol{x}, s)= & \frac{\cos \theta^{i}}{Z_{f}}\left(e^{-s \frac{x}{c_{f}} \cos \theta^{i}}\right. \\
& \left.-R\left(s, \theta^{i}\right) e^{s \frac{x}{c_{f}} \cos \theta^{i}}\right) e^{-s \frac{y}{c_{f}} \sin \theta^{i}}
\end{aligned}\right.
$$

where $R\left(s, \theta^{i}\right)$ is the Laplace transform of the reflection kernel of interest, $Z_{f}=\rho_{f} c_{f} \approx 418 \mathrm{~kg} \cdot \mathrm{m}^{-2} \cdot \mathrm{s}^{-1}$ is the characteristic impedance of the material, and $V_{1}(\boldsymbol{x}, s)$ is the $x$ component of the velocity. Inside the material, the fields are expressed, for $0 \leq x<+\infty$, as

$$
\left\{\begin{array}{l}
P_{2}(\boldsymbol{x}, s)=A(s) e^{-s \frac{x}{c_{m}} \cos \theta^{m}} e^{-s \frac{y}{c_{m}} \sin \theta^{m}} \\
V_{2}(\boldsymbol{x}, s)=\frac{\cos \theta_{1}}{Z_{m}} A(s) e^{-s \frac{x}{c_{m}} \cos \theta^{m}} e^{-s \frac{y}{c_{m}} \sin \theta^{m}}
\end{array}\right.
$$

where $A$ is an unknown parameters, $\theta^{m}$ is the refracted wave angle in the porous material, relative to its normal, and $V_{2}(\boldsymbol{x}, s)$ is the $x$ component of the velocity. The phase velocity in the equivalent fluid material is defined as $c_{m}=c_{f} / \sqrt{\alpha(s) \beta(s)}$, while the characteristic impedance of the material is given by $Z_{m}=Z_{f} \sqrt{\frac{\alpha(s)}{\beta(s)}}$.

Using the boundary conditions of Eq. 7, one obtains the equation system

$$
1+R\left(s, \theta^{i}\right)=A(s), 1-R\left(s, \theta^{i}\right)=\phi \frac{Z_{f} \cos \theta^{m}}{Z_{m} \cos \theta^{i}} A(s) .
$$

Solving the system 13 yields the reflection coefficient $R\left(s, \theta^{i}\right)$ as

$$
\begin{gathered}
R\left(s, \theta^{i}\right)=\frac{1-E(s)}{1+E(s)}, \quad E(s)=\frac{\cos \theta^{m}}{\cos \theta^{i}} \phi \sqrt{\frac{\beta(s)}{\alpha(s)}}, \\
\cos \theta^{m}=\sqrt{1-\frac{\sin ^{2} \theta^{i}}{\alpha(s) \beta(s)}},
\end{gathered}
$$


and where the Snell-Descartes law has been used. Using the inverse Laplace transform to go back into the time domain, the reflection coefficient at the first interface described in Eq. 14 acts as an operator whose expression is:

$$
\begin{aligned}
\tilde{R}\left(t, \theta^{i}\right)= & \frac{1-E_{0}}{1+E_{0}} \delta(t)+\frac{2 E_{0} \sqrt{\eta / \rho_{f}}}{\left(\alpha_{\infty}-\sin ^{2} \theta^{i}\right)\left(1+E_{0}\right)^{2}} \\
& \times\left(\frac{\alpha_{\infty}-2 \sin ^{2} \theta^{i}}{\Lambda}-\frac{\alpha_{\infty}(\gamma-1)}{\Lambda^{\prime} \sqrt{\mathrm{Pr}}}\right) \frac{H(t)}{\sqrt{\pi t}}
\end{aligned}
$$

with

$$
E_{0}=\frac{\phi \sqrt{\alpha_{\infty}-\sin ^{2} \theta^{i}}}{\alpha_{\infty} \cos \theta^{i}}
$$

Once the reflection operator is known, one can convolve the incident signal with it in order to yield the numerical reflected signal in the time domain, for a given incident angle $\theta^{i}$, which can in turn be compared to the experimental reflected signal, allowing one to carry on with the inverse method, as presented in Section III. Anticipating Section II.C, let us consider a porous material (P1) with certain properties (given in Table 1). An incident transient pressure wave $p^{i}$ impinges on the material and is partly reflected. In Figure 2, we plot the experimental incident signal, the numerically calculated reflected signal and the reflection operator used in the convolution.

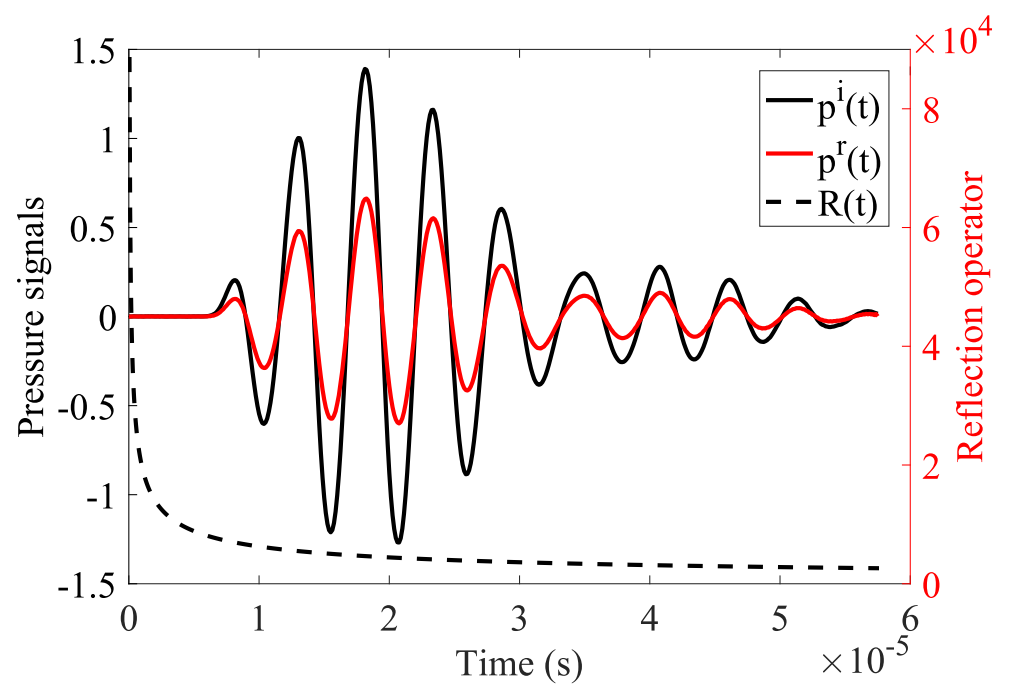

Figure 2: (Color online) Time domain representation of reflected pressure signals (left axis) and reflection operator (right axis)

\subsection{Critical angle at oblique incidence}

An important property of the reflection coefficient can be identified by first simplifying Eq. 15 as

$$
\tilde{R}\left(t, \theta^{i}\right)=\tilde{R}_{0}\left(t, \theta^{i}\right)+\tilde{R}_{1}\left(t, \theta^{i}\right),
$$

with

$$
\tilde{R}_{0}\left(t, \theta^{i}\right)=\frac{1-E_{0}}{1+E_{0}} \delta(t)
$$

and

$$
\begin{aligned}
\tilde{R}_{1}\left(t, \theta^{i}\right)= & \frac{2 E_{0} \sqrt{\eta / \rho_{f}}}{\left(\alpha_{\infty}-\sin ^{2} \theta^{i}\right)\left(1+E_{0}\right)^{2}} \\
& \times\left(\frac{\alpha_{\infty}-2 \sin ^{2} \theta^{i}}{\Lambda}-\frac{\alpha_{\infty}(\gamma-1)}{\Lambda^{\prime} \sqrt{\operatorname{Pr}}}\right) \frac{H(t)}{\sqrt{\pi t}}
\end{aligned}
$$


where $\tilde{R}_{1}$ corresponds to the dispersive part of the reflection coefficient. One observes that $\tilde{R}_{1}$ can be canceled if there exists a critical angle $\theta_{c, 1}$ verifying

$$
\sin ^{2} \theta_{c, 1}=\frac{1}{2} \alpha_{\infty}\left(1-\frac{\Lambda}{\Lambda^{\prime}} \frac{\gamma-1}{\sqrt{\operatorname{Pr}}}\right)
$$

Measuring such an angle can be done by detecting, for instance, a change in the sign of the imaginary part of the total reflection coefficient, which can be readily measured by first transforming the incident and reflected signals in the frequency domain via an fast Fourier transform, and calculating the ratio between the two signals. The higher the tortuosity and the lower the ratio $\Lambda / \Lambda^{\prime}$, the higher the critical angle becomes. This is the first time that this relationship has been highlighted, and its knowledge used in inverse identification methods. There also exists an angle $\theta_{c}$ for which $\tilde{R}\left(t, \theta_{c}\right)=0$, as was shown initially in [28]. While its expression is time dependent, estimating its value numerically can be done quickly. This angle will not be used in the inverse problem, as it was not detected experimentally. In the same fashion, an angle $\theta_{c, 0}$ for which $\tilde{R}_{0}\left(t, \theta_{c, 0}\right)=0$ exists and is relatively close to $\theta_{c}$. Again, its value was not measured experimentally for the cases at hand and thus its expression was not used further during the inference.

\subsection{Illustrations of the direct problem}

For completeness, let us consider 3 different numerically generated porous materials (P1, P2 and P3) and display their reflection coefficient and critical angles (properties were chosen close to the ones inferred later on). The material properties are summarized in Table 1, along with the critical angle $\theta_{c, 1}$ for which $\tilde{R}_{1}$ cancels out, and the critical angle $\theta_{c}$ for which the full reflection cancels out, at $190 \mathrm{kHz}$. The spread of these two values is increased for low porosity materials that typically display a higher reflection coefficient. We now display the full reflection coefficient against the

\begin{tabular}{|c|c|c|c|c|c|c|}
\hline Name & $\phi$ & $\alpha_{\infty}$ & $\Lambda(\mu \mathrm{m})$ & $\Lambda^{\prime}(\mu \mathrm{m})$ & $\theta_{c, 1}\left(^{\circ}\right)$ & $\theta_{c}\left(^{\circ}\right)$ \\
\hline \hline P1 & 0.5 & 1.7 & 30 & 90 & 57.8 & 74.5 \\
\hline P2 & 0.8 & 1.5 & 50 & 150 & 52.6 & 62.7 \\
\hline P3 & 0.99 & 1.04 & 200 & 400 & 39.0 & 46.6 \\
\hline
\end{tabular}

Table 1: Synthetic porous media: summary of their properties

angle of incidence at $190 \mathrm{kHz}$ for all three materials studied, in Figure 3 (left). The dispersive part of the reflection coefficient, $R_{1}\left(s, \theta^{i}\right)$, is also displayed in Figure 3 (right). When looking at low values of incidence angles, the dispersive contribution is more important for materials of type P1 and P2 (associated with high flow resistivities). This result is in agreement with our initial comments that the dispersive correction of the reflection is greater for resistive, low characteristic length materials. However, this is surprisingly not the case anymore for high values of the incidence angle, for which $R_{1}\left(s, \theta^{i}\right)$ takes more important values in the case of material P3 (associated with low flow resistivities). This raises the question of where (in terms of the incident angle) one should observe a given material in order to optimally determine its parameters. Our examples suggest that highly resistive materials should be observed at low angles of incidence, while low resistivity materials could yield better identification results when observed at higher incidence angles, provided that the added uncertainty stemming from grazing angles of incidence remains low. In Figure 4 , we show a decomposition of the reflected waves for the three materials considered, at an angle where the dispersive nature of the material is the most marked: using Figure 3(right) yields an angle of $0^{\circ}$ for materials $\mathrm{P} 1$ and $\mathrm{P} 2$, and an angle of $80^{\circ}$ for material P3. This decomposition consists in the reflected wave associated with the instantaneous response of the material (convolution of the incident wave with $\tilde{R}_{0}\left(t, \theta^{i}\right)$ ) and the dispersive part of the reflection (convolution of the incident wave with $\left.\tilde{R}_{1}\left(t, \theta^{i}\right)\right)$, the sum of which yields, by linearity of the convolution operator, the full reflected wave (associated with the convolution of the incident wave with $\tilde{R}\left(t, \theta^{i}\right)$ ). As expected, the contribution of the dispersive nature of porous media is significant and has to be taken into 

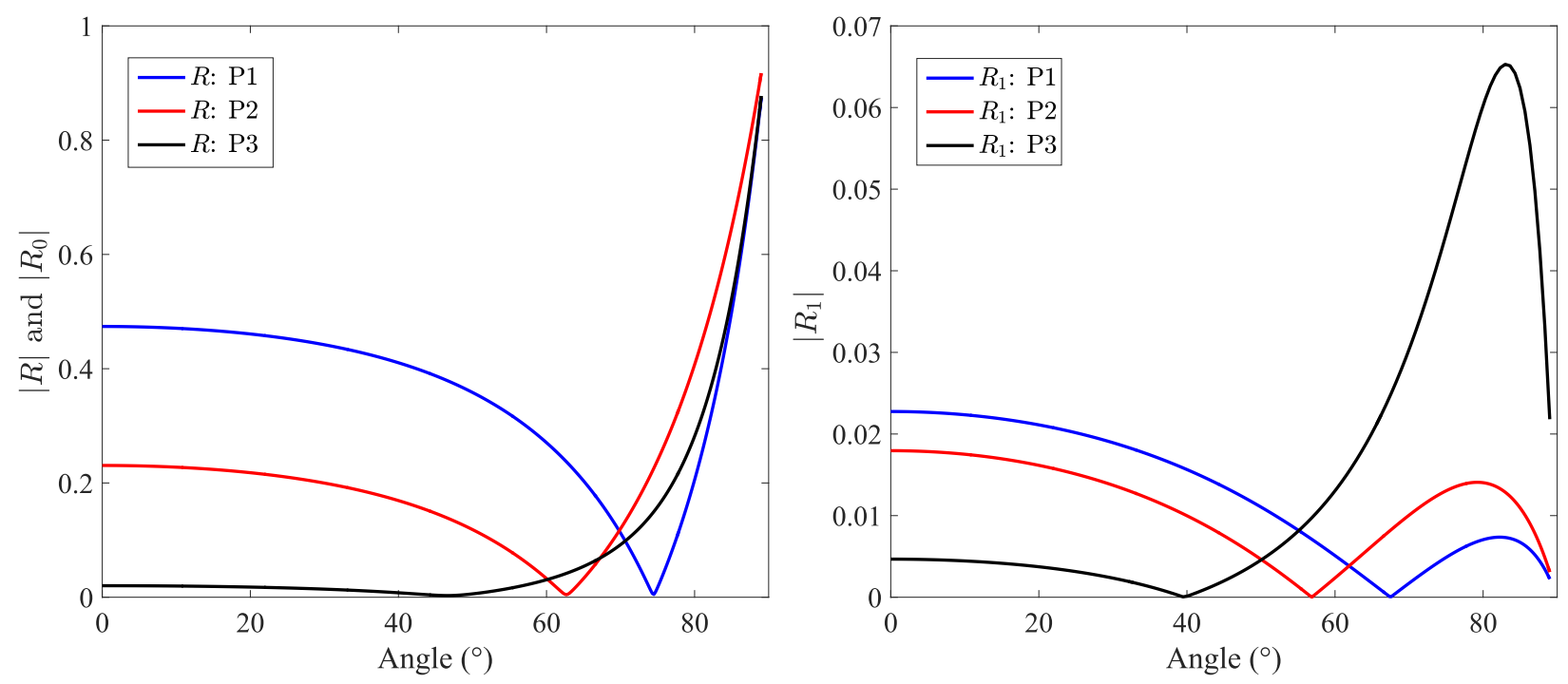

Figure 3: (Color online)For the three synthetic materials P1, P2 and P3, at $190 \mathrm{kHz}$. Left: Absolute value of the total reflection coefficient $\left|R\left(s, \theta^{i}\right)\right|$ as a function of the incident angle. Right: Absolute value of the scattering part of the reflection coefficient $\left|R_{1}\left(\theta^{i}\right)\right|$

account, even for low resistivity materials, when high angles of incidence are concerned. This result has not been mentioned in the literature before, to the best of the authors' knowledge, and opens the way for new characterization methods, focusing the observation on regions of interest depending on prior knowledge on the porous material nature and pore scales (which is often a readily available piece of information). Since $\tilde{R}_{1}\left(t, \theta^{i}\right)$ is a significant contribution to the reflection and depends on the parameters $\phi, \alpha_{\infty}, \Lambda, \Lambda^{\prime}$ to be identified, we can now proceed with the inference method.
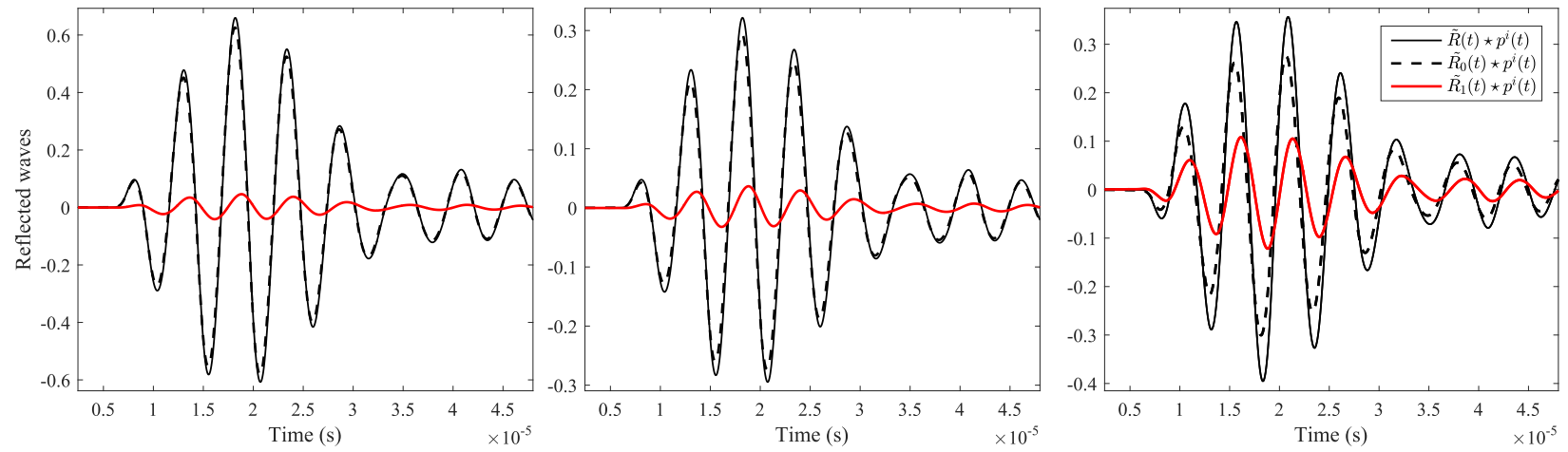

Figure 4: (Color online) Reflected waves in the time domain associated with three different reflection operators: $\tilde{R}, \tilde{R}_{0}, \tilde{R}_{1}$. For material P1 at angle of incidence $\theta^{i}=0^{\circ}$ (left), material P2 at angle of incidence $\theta^{i}=0^{\circ}$ (middle), and material P3 at angle of incidence $\theta^{i}=80^{\circ}$ (right). The angles are chosen according to Figure 3 (right), corresponding to the maximum of $\tilde{R}_{1}$.

\section{Statistical inference}

In the context of inverse problems for parameter estimation, statistical inference in the Bayesian framework consists in recasting parameters of interest as random variables associated with probability densities encompassing established information, or new information collected through measurements. The solution of such an inverse problem is no longer a single value for the estimated parameters of interest, but their posterior probability density [29, Chap. 8]. A good review on inverse problem theory can be found in [30]. 


\subsection{Bayes's theorem}

Statistical inverse problems rely on information from both the observed experimental data $\boldsymbol{d}^{\exp }$ and the prior knowledge on the parameters $\boldsymbol{m}$. In the present article, $\boldsymbol{d}^{\exp }$ encompasses all the measured reflected signals in the time domain, and is thus represented by a matrix of size $N_{d} \times K$, where $N_{d}$ is the number of observed discrete time steps and $K$ is the number of different incident angles considered. The parameters of interest are $\boldsymbol{m}=\left(\phi, \alpha_{\infty}, \Lambda, \Lambda^{\prime}\right)$. These two states of information are combined to express our degree of knowledge about the true values of $\boldsymbol{m}$, improved by the information contained in the observation $\boldsymbol{d}^{\exp }$. We define a marginal posterior probability density $\pi\left(\boldsymbol{m} \mid \boldsymbol{d}^{\text {exp }}\right)$ for the model parameters $\boldsymbol{m}$, given a set of observable parameters $\boldsymbol{d}^{\text {exp }}$. Using Bayes' theorem, this becomes

$$
\pi\left(\boldsymbol{m} \mid \boldsymbol{d}^{\exp }\right)=\frac{\pi\left(\boldsymbol{d}^{\exp } \mid \boldsymbol{m}\right) \pi(\boldsymbol{m})}{\pi\left(\boldsymbol{d}^{\exp }\right)}
$$

where $L(\boldsymbol{m}) \equiv \pi\left(\boldsymbol{d}^{\exp } \mid \boldsymbol{m}\right)$ is the likelihood function, $\pi(\boldsymbol{m})$ is the prior density, and $\pi\left(\boldsymbol{d}^{\exp }\right)$ is the Bayesian evidence. Prior and posterior densities represent the state of knowledge, or degree of belief we have on the parameters, before and after having observed a certain piece of data $\boldsymbol{d}^{\text {exp }}$, respectively. The posterior probability $\pi\left(\boldsymbol{m} \mid \boldsymbol{d}^{\exp }\right)$ reflects all the information inferred on $\boldsymbol{m}$, conditional on the observation $\boldsymbol{d}^{\exp }$, and is the quantity to be found. The evidence $\pi\left(\boldsymbol{d}^{\exp }\right)$ represents the probability of the experimental data being generated by the given parameters and models; the evidence is generally a term difficult to evaluate numerically: its calculation can be conveniently circumvented by the MCMC method detailed in Subsection III.D. The aforementioned terms are detailed in the following.

\subsection{Likelihood}

The information on the physical correlations between $\boldsymbol{m}$ and $\boldsymbol{d}^{\exp }$ as predicted by a physical model, here a convolution between incident signal and reflection operator, is represented by a deterministic forward operator $\boldsymbol{G}$, and a noise $\boldsymbol{\epsilon}$ accounting for our uncertainty relative to the experiment, at each observed time step, and for all angles. Thus, $\boldsymbol{\epsilon}$ is of size the size of $\boldsymbol{d}^{\text {exp }}$. For each incidence angle $\theta_{k}$,

$$
\boldsymbol{d}_{k}^{\exp }=\boldsymbol{G}\left(\boldsymbol{m}^{*}, \theta_{k}\right)+\boldsymbol{\epsilon}_{\boldsymbol{k}}
$$

with $\boldsymbol{m}^{*}$ unknown and where the components of $\boldsymbol{\epsilon}_{\boldsymbol{k}}$ are assumed independent and identically distributed random variables of zero mean Gaussian density $\pi_{\epsilon}$. The forward operator is represented in the discrete-time way by noting $\boldsymbol{G}\left(\boldsymbol{m}, \theta_{k}\right)=\left(G_{1}\left(\boldsymbol{m}, \theta_{k}\right), \ldots G_{j}\left(\boldsymbol{m}, \theta_{k}\right), \ldots, G_{N_{d}}\left(\boldsymbol{m}, \theta_{k}\right)\right)$, where $N_{d}$ is the number of time-steps observed in the experimental reflected signal, and with

$$
G_{j}\left(\boldsymbol{m}, \theta_{k}\right)=\int_{0}^{t_{j}} \tilde{R}\left(\boldsymbol{m}, \tau, \theta_{k}\right) p^{i}\left(\boldsymbol{x}_{\text {meas }}, t_{j}-\tau\right) \mathrm{d} \tau,
$$

where the dependency on the parameters $\boldsymbol{m}$ has been made explicit in the writing of the reflection kernel. The measurement location is noted by $\boldsymbol{x}_{\text {meas }}$, and $t_{j}$ is the $j^{\text {th }}$ observed time step. The $j^{\text {th }}$ component of $\boldsymbol{d}^{\text {exp }}$ becomes, for the $k^{\text {th }}$ incident angle:

$$
d_{j, k}^{\exp }=G_{j}\left(\boldsymbol{m}, \theta_{k}\right)+\epsilon_{j, k},
$$

The likelihood of an observation conducted with a given incident angle $\theta_{k}$ takes the form

$$
L\left(\boldsymbol{m}, \theta_{k}\right)=\pi_{\epsilon}\left(\boldsymbol{d}_{k}^{\exp }-\boldsymbol{G}\left(\boldsymbol{m}, \theta_{k}\right)\right)
$$

which reads, assuming $\pi_{\epsilon}$ follows a zero mean Gaussian distribution,

$$
L\left(\boldsymbol{m}, \theta_{k}\right)=\prod_{j=1}^{n_{d}} \frac{1}{\sqrt{2 \pi \sigma_{j, k}^{2}}} \exp \left(-\frac{\left|d_{j, k}^{\exp }-G_{j}\left(\boldsymbol{m}, \theta_{k}\right)\right|^{2}}{2 \sigma_{j, k}^{2}}\right),
$$




\begin{tabular}{|c|c|c|c|c|}
\hline Parameter & $\phi$ & $\alpha_{\infty}$ & $\Lambda(\mu \mathrm{m})$ & $\Lambda^{\prime} / \Lambda$ \\
\hline \hline Prior Min & 0.2 & 1.0 & 1.0 & 1.0 \\
\hline Prior Max & 0.99 & 3.0 & 1000 & 10 \\
\hline
\end{tabular}

Table 2: Prior boundaries

where $\sigma_{j, k}^{2}$ denotes the noise variance associated to a time step and an incident angle, and is either determined experimentally or set a priori, for each signal. The larger the value of $\sigma_{j, k}^{2}$, the larger the estimated uncertainty on the observation. The value of the standard deviation $\sigma_{j, k}$ is taken in this work to $2 \%$ of the absolute value of the considered experimental reflected signal, to encompass our uncertainty in the acquisition chain and the experimental conditions (temperature, pressure, etc...). For numerical reasons, the logarithm of the likelihood is used instead of Eq. 26. The total log-likelihood is then obtained as

$$
\log (L(\boldsymbol{m}))=\sum_{k=1}^{K} \log \left(L\left(\boldsymbol{m}, \theta_{k}\right)\right),
$$

where $K$ is the number of incident angles used. Similar to classical inverse problems where a cost function has to be minimized in order to retrieve the parameters of interest, one can view the likelihood as the probabilistic equivalent of such a quantity, effectively measuring the probability of the experience being observed, for a given set of parameters.

\subsection{Prior model}

The prior probability $\pi(\boldsymbol{m})$ contains the information obtained on the model parameters independently of the experimental data used during the inference. It reflects the user's prior insight into the problem, generalizing the classical constraints used during deterministic inversion, assigning weights to the more probable values. When no prior information is available, a wide-range, non-informative improper homogeneous density represents well one's ignorance and merely sets boundaries for each parameter, as in classical deterministic inverse problems. In the present article, we first assume prior independence among the unknown parameters, setting

$$
\pi(\boldsymbol{m})=\prod_{i=1}^{n_{m}} \pi_{i}\left(m_{i}\right)
$$

In the absence of observation, there is no known correlation between the parameters. As no statistical information on the parameters was accessible prior to the inference, a uniform probability $\pi_{i}\left(m_{i}\right) \sim \mathcal{U}\left(m_{i, \min }, m_{i, \max }\right)$ is assumed for the priors, with $m_{i, \min }$ and $m_{i \text {,max }}$ the boundaries, given in Table 2. To further constrain the problem, when possible, one can include a known correlation between the parameters, using the approximate knowledge of the critical angle defined in Eq. 20 . As this information is gained through the observation of the signals in the frequency domain, it can still be seen as a prior (independent) knowledge, since the inference is performed on the reflected signals in the time domain. This method is applied for the inference of material M2 in Section IV.C.

\subsection{MCMC: posterior sampling}

As the posterior probability density defined in Eq. 21 is only known up to a normalizing constant, one still needs to devise a technique to draw samples directly from the posterior distribution, without having to calculate the evidence, which is especially arduous in high-dimensional parameter spaces. To address this issue, a technique called Markov Chain Monte Carlo (MCMC) was exploited.

The central idea behind MCMC is to draw samples directly from the posterior distribution without having to calculate the normalizing factor, by designing a random-walk algorithm that 
explores the posterior density and moves preferentially towards regions of high probability densities, efficiently exploring the area of interest and creating a Markov chain whose stationary distribution is the distribution of interest [31. This is done with a Metropolis-Hasting algorithm [32, 33], consisting in an acceptance/rejection step that creates the transition kernel of a Markov chain converging to the posterior distribution. The MCMC strategy is coupled with a Differential Evolution algorithm [34]: multiple chains are run in parallel and learn from each other through a mutation operator, thus solving the problems of selecting a direction and a scale for the jump from the current point to the proposal point [35, 36]. In the present study, the latest improvements on MCMC techniques were integrated, making use of multi-try sampling from past states, snooker updates and self-adaptive randomized subspace sampling. The full details on this method, coined MT_DREAM_ZS, are given in [37] and the references therein.

In the present work, 3 chains were used on which $10^{5}$ iterations were performed, with the first $10 \%$ of each chain being discarded as burn-in to allow decorrelation with the initial samples. The chains were initialized by sampling from the prior's support. Convergence was checked a posteriori, through a Gelman-Rubin diagnostic [38] and a visual check of the chain samples.

\section{Results}

In this section, three widely different materials are tested and their properties inferred. The first material, M1, consists in a random packing of spheres and is highly resistive; material M2 is a resistive foam and material M3 is a classical foam used as a sound absorbing material, of low estimated resistivity. Due to its low resistivity and the low values of incident angles, the reflected wave amplitudes of M3 are lower than for the two other materials, and it is interesting to test the limits of the present method in such a case. For the first and third materials, no critical angle $\theta_{c, 1}$ was observed; for the second material, it was estimated. For all materials, the inference is summarized through the display of posterior probability density functions (pdfs) of all parameters, along with joint-pdfs to reveal the correlations between parameters. These were evaluated from the chain samples through a kernel density estimation. Other experimental results, obtained with reference methods developed in [39, 40, 16, 41, are displayed as well. The Maximum A Posteriori (MAP) estimator was chosen as the reference probabilistic estimator in this study, since it is closest in meaning to the estimates obtained through usual deterministic methods, related to a maximum likelihood estimation. The MAP $\boldsymbol{m}_{\mathrm{MAP}}$ estimate is defined as

$$
\boldsymbol{m}_{\mathrm{MAP}}=\arg \max _{\boldsymbol{m}} \pi\left(\boldsymbol{m} \mid \boldsymbol{d}^{\mathrm{exp}}\right)
$$

\subsection{Experimental procedure}

Experiments were performed in air with two broadband Ultran NCT202 transducers with a $190 \mathrm{kHz}$ center frequency and $6 \mathrm{~dB}$ bandwidth extending from 150 to $230 \mathrm{kHz}$. The associated wavelength is $\lambda=c_{f} / f \approx 1.8 \mathrm{~mm}$, which remains larger than the pore sizes of all the materials considered in this study. An optical goniometer was used to position the transducers. Uncertainties on the angle measurements are not considered in the present work. Pulses of $400 \mathrm{~V}$ amplitude were provided by a 5052PR Panametrics pulser/receiver. The signals received were amplified to $90 \mathrm{~dB}$ and filtered above $1 \mathrm{MHz}$ to avoid high frequency noise. Electronic interference was removed by 1000 acquisition averages. Measuring the reference incident signal was done with the help of a rigid metal plate acting as an acoustic mirror. The experimental setup is shown in Figure 5.

\subsection{M1 identification: sphere packing}

The waves reflected from material M1 were measured at 3 different angles of incidence $\left(13.5^{\circ}, 17^{\circ}\right.$, $66.6^{\circ}$ ). Figure 6 shows the posterior marginal probability density functions of each parameter (diagonal elements) and the 2D scatter-plot obtained from the Markov Chains (off-diagonal elements) 


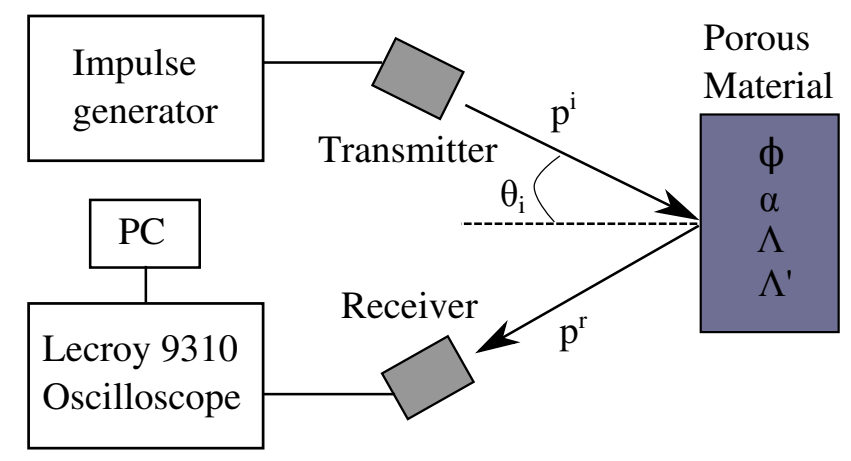

Figure 5: Simplified schematic of the experimental apparatus

inferred on the material M1 properties. The 4 colored curves on the scatter plots each delimit $20 \%$ of the scattered points, thus representing credibility intervals of 20, 40, 60 and $80 \%$, respectively. Such an interval is interpreted as a percentage probability that the true value of a given set of 2 parameters falls within the credible region.

These results are summarized in Table 3 where the MAP and standard deviation of each parameter are reported. Results obtained in [16] using classical identification techniques on the same material are also recalled for reference. These reference measurements did not account for the dispersive contribution to the reflection operator, thus explaining the difference between obtained values. Interestingly, the porosity remains quite close in both cases. While $\phi, \alpha_{\infty}$ and $\Lambda$ densities have a Gaussian shape, the density of $\Lambda^{\prime} / \Lambda$ is less informative, indicating the asymmetric loss of sensitivity relative to the likelihood function when $\Lambda^{\prime}$ increases (seen by the flattening of the pdf). Note that the extreme limit imposed by the prior $\Lambda^{\prime} / \Lambda=10$ is reached for this material, and that a larger boundary would have extended the density support.

A joint-pdf of approximate elliptic shapes such as the ones between $\phi, \alpha_{\infty}$ and $\Lambda$ in Fig. 6 denotes a positive correlation between parameters. This indicates that an increase in the estimation of one parameter results in an increase in the correlated parameter. These correlations need to be taken with caution, as they do not necessarily denote a physical correlation between the parameters. For instance, a material with very high porosity tends to have lower tortuosity, which is opposite to what could be understood from the joint-pdf. These correlations stem from the modeling structure and the experimental observations used in the inference. Correlations between parameters are expected to be strong, due to their association in the reflection coefficient expression, in Eq. 15 . For example, if one looks at the expression of $E_{0}$ (Eq. 16), which intervenes in the definition of $\tilde{R}_{0}$, one sees that at $\theta_{i}=0, E_{0}=\phi / \sqrt{\alpha_{\infty}}$. A simultaneous increase in both $\phi$ and $\alpha_{\infty}$ could then leave $E_{0}$ unchanged, which in turn would result is a sensibly similar likelihood function, thus explaining the positive correlations between those two parameters during Bayesian inference process. Taking into account $\tilde{R}_{1}$ would change these correlations, but the idea remains the same.

Should a more informative prior be stipulated for the porosity, the posterior marginal probability density of $\phi$ would be shifted towards the prior, and $\alpha_{\infty}$ and $\Lambda$ would be shifted in the same direction (increase or decrease). In the case where no prior is used, the Bayesian inference is called objective [42]. An objective inference allows to learn about correlations between parameters, stemming from the modeling structure or the experimental observation. Knowing these correlations, one can then anticipate the influence of setting a more informative prior on a certain parameter.

In second level Bayesian inference [42], the prior modeling itself can enter the inference process, resulting in extra parameters in the identification, and model selection. In the present study, only the first level Bayesian inference (model calibration) was considered. 


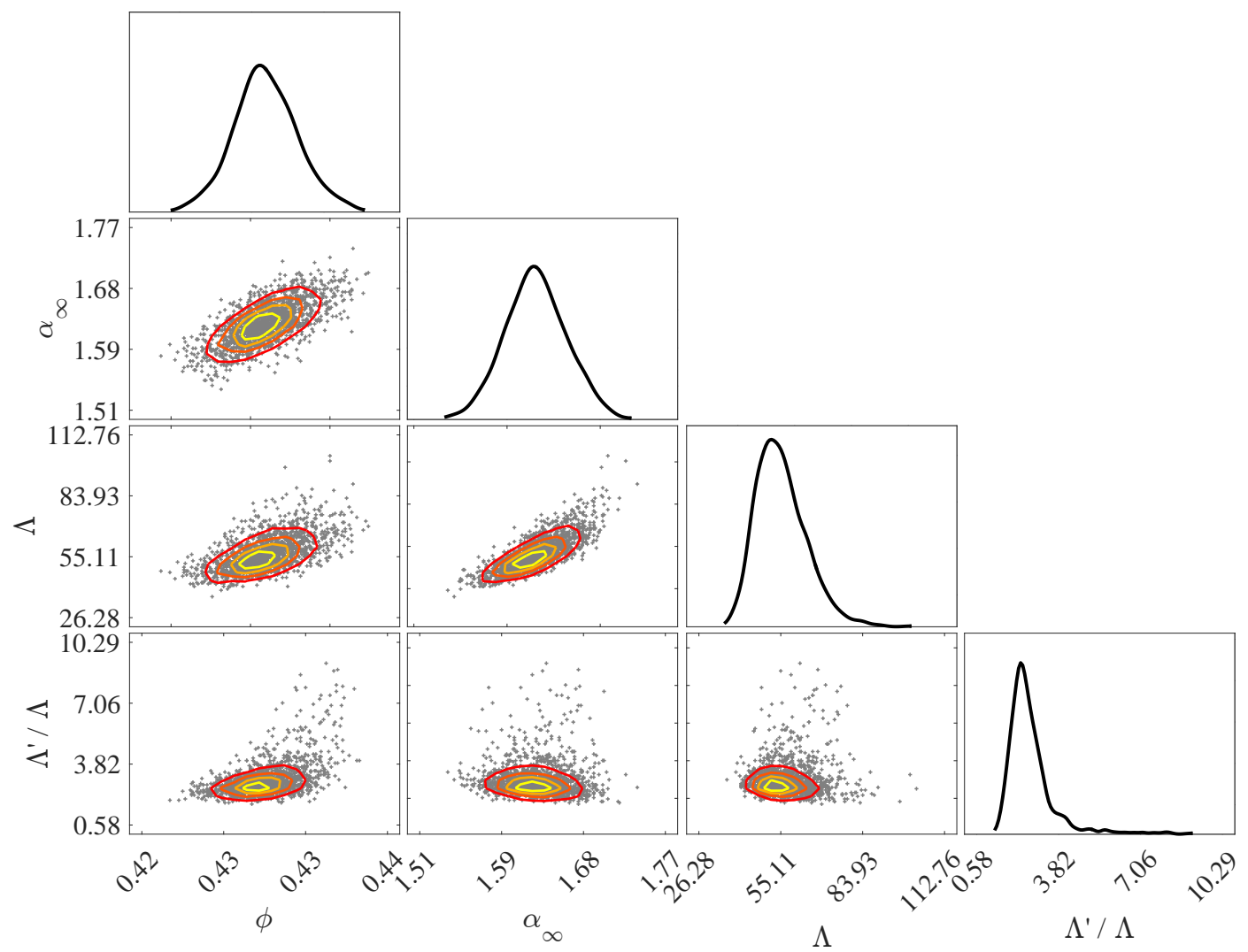

Figure 6: (Color online) M1 inference pdfs (the graphs on the diagonal) and joint-pdfs (the off-diagonal scatter plots) 

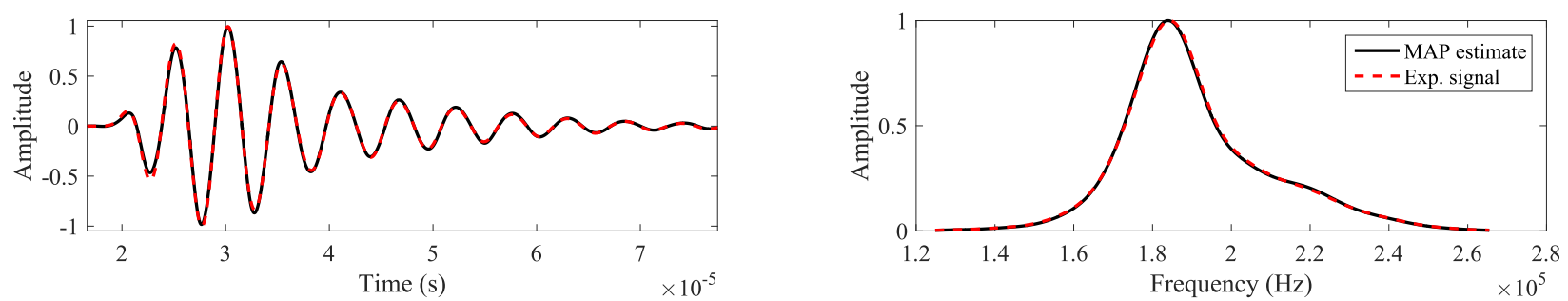

Figure 7: (Color online) Normalized experimental signals : _ _ and normalized MAP estimate: — at $\theta_{i}=66^{\circ}$. Left: normalized time signals. Right: normalized frequency spectrum.

\begin{tabular}{|c|c|c|c|c|}
\hline Parameter & $\phi$ & $\alpha_{\infty}$ & $\Lambda(\mu \mathrm{m})$ & $\Lambda^{\prime} / \Lambda$ \\
\hline \hline Reference values & 0.44 & 1.87 & $\mathrm{~N} / \mathrm{A}$ & $\mathrm{N} / \mathrm{A}$ \\
\hline Inverted MAP value & 0.43 & 1.63 & 52.9 & 2.6 \\
\hline Standard deviation & 0.002 & 0.03 & 8.1 & 0.88 \\
\hline
\end{tabular}

Table 3: M1 inference summary (N/A: non-available). Reference results are obtained with methods developed in [16].

The signals calculated using the Markov chain samples are compared with the experimental reflected signals in Figure 7 for a particular incident angle used in the inference. The Fourier transform is also used to compare the spectral density of calculated and experimental signals for the same two angles. Signals are normalized using the maximum value of the experimental signal. The remaining small discrepancies between signals can be explained in the following two ways: the rigid skeleton hypothesis may not be adequate in the case of high amplitude waves for sphere packing materials such as this one, due to a movement between the spheres. Furthermore, the geometry of the representative pore itself is somewhat out of the scope of the JCA model, where it is assumed that no constriction is present between pores. Due to the sphere curvatures, it is possible that the model corrections of Pride [43] are necessary to represent more accurately the wave propagation in such a material. This is left for a future study.

\subsection{M2 identification: high resistivity foam}

The waves reflected from material M2 were measured at 4 different angles of incidence $\left(0^{\circ}, 23^{\circ}\right.$, $39.7^{\circ}, 49.8^{\circ}$ ). Figure 8 shows the pdfs (diagonal elements) and joint-pdfs (extra-diagonal elements) inferred on the material M2 properties. These results are summarized in Table 4 where the MAP and standard deviation of each parameter are reported. Results obtained using classical identification techniques are also recalled for reference. The small discrepancies between reference and MAP values can be attributed to a lower sensitivity of the porosity in the transmission mode at high frequencies [27]. All the parameters display a pdf of Gaussian shape. Once again, elliptic joint-pdfs denote the correlations between parameters, stemming from the modeling used.

\begin{tabular}{|c|c|c|c|c|}
\hline Parameter & $\phi$ & $\alpha_{\infty}$ & $\Lambda(\mu \mathrm{m})$ & $\Lambda^{\prime} / \Lambda$ \\
\hline \hline Reference values & 0.75 & 1.45 & 50 & $2-3$ \\
\hline Inverted MAP value & 0.80 & 1.44 & 45.4 & 2.3 \\
\hline Standard deviation & 0.003 & 0.02 & 3.2 & 0.22 \\
\hline
\end{tabular}

Table 4: M2 inference summary. Reference results are obtained with methods developed in [39, 40, 41].

The results presented here take into account prior information obtained on the critical angle defined in Eq. 20, estimated between $\theta_{i}=39.7^{\circ}$ and $\theta_{i}^{\prime}=49.8^{\circ}$. Indeed, as can be seen in Figure 9 . the phase of the experimental coefficient is seen to become negative between these two values. The 


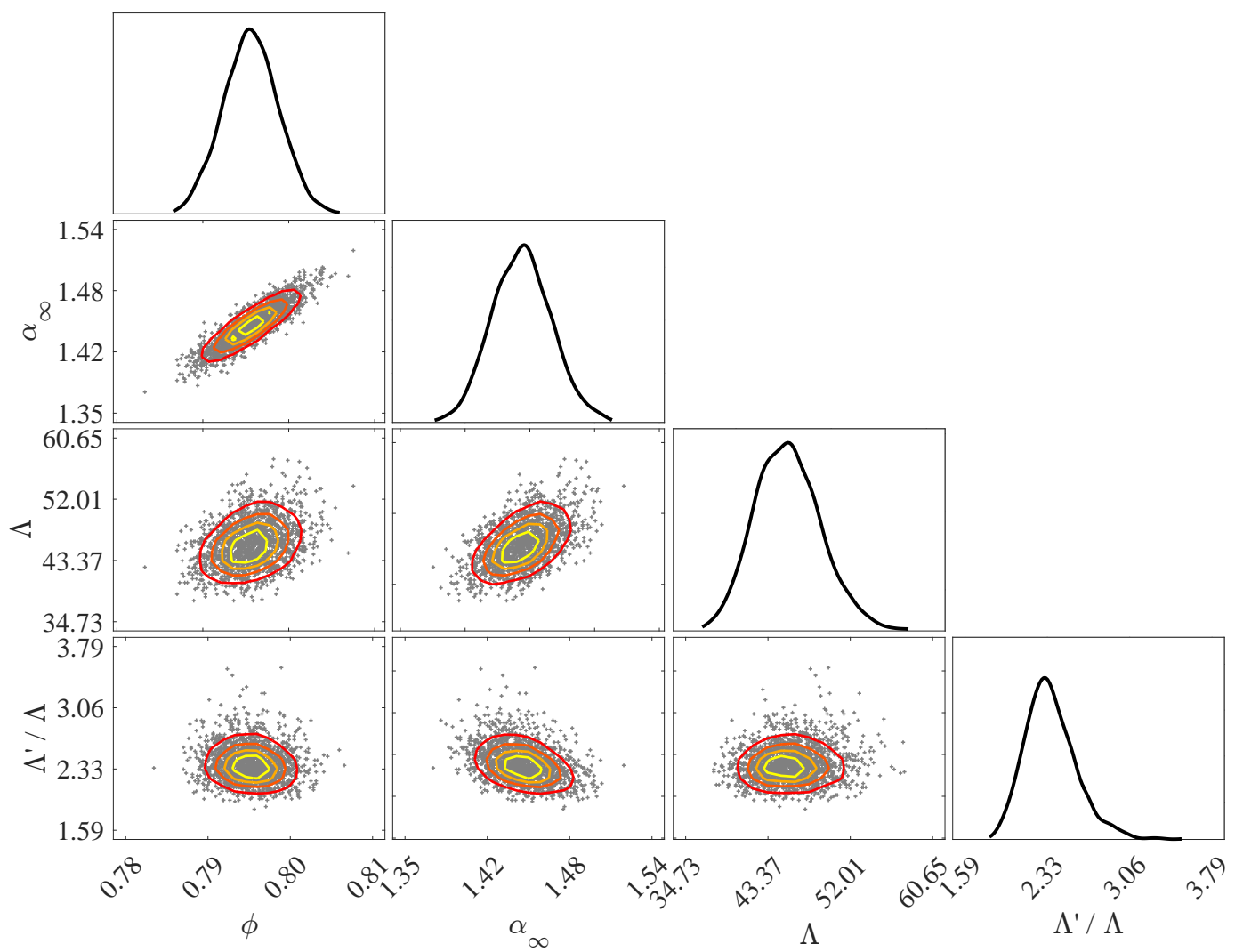

Figure 8: (Color online) M2 inference pdfs (the graphs on the diagonal) and joint-pdfs (the offdiagonal scatter plots) 


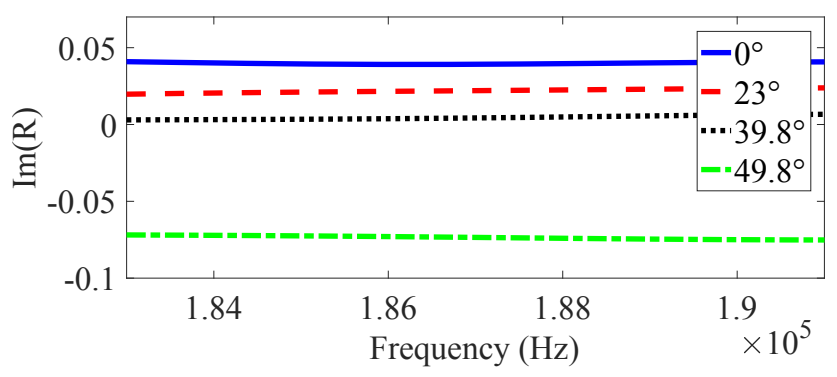

Figure 9: (Color online) Imaginary part of the reflection coefficient for material M2, at four different incident angles.
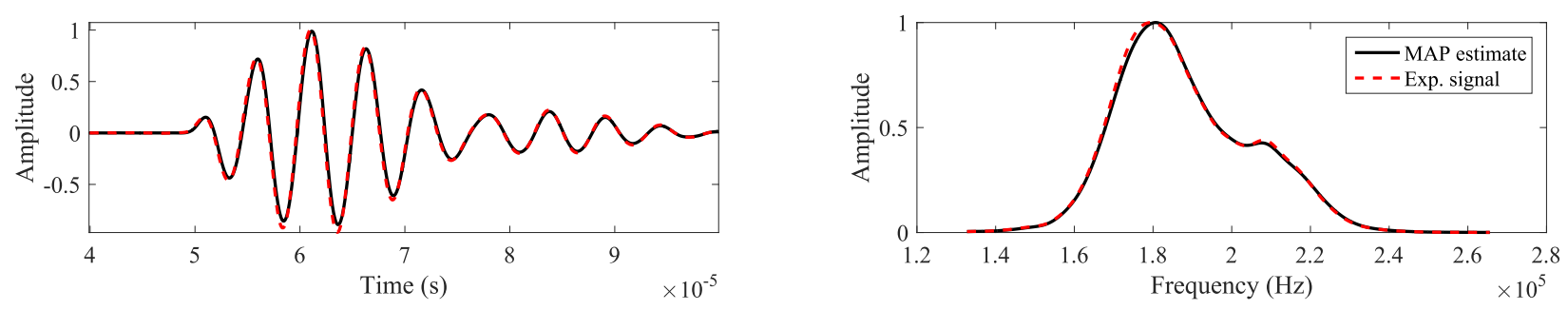

Figure 10: (Color online) Normalized experimental signals : _ _ and normalized MAP estimate: — at $\theta_{i}=23^{\circ}$. Left: normalized time signals. Right: normalized frequency spectrum.

prior density function is updated as

$$
\pi(\boldsymbol{m}) \times \pi_{\theta_{c}}\left(a_{\theta c}\right) \leftarrow \pi(\boldsymbol{m})
$$

where $a_{\theta c}=1 / 2 \alpha_{\infty}\left(1-\left(\Lambda / \Lambda^{\prime}\right)(\gamma-1) / \sqrt{\operatorname{Pr}}\right)$ and $\pi_{\theta_{c}}$ is a normal distribution centered on $\left(\sin ^{2} \theta_{i}+\right.$ $\left.\sin ^{2} \theta_{i}^{\prime}\right) / 2$ with standard deviation $\left(\sin ^{2} \theta_{i}-\sin ^{2} \theta_{i}^{\prime}\right) / 6$. The standard deviation is chosen so as to get $99 \%$ of the probabilistic weight between the observed boundaries. This choice is made in light of our lack of certainty regarding the exactness of relation 20, which, in particular, does not hold any longer when the Pride correction [43] is taken into account.

To further validate the inference, the signals calculated using the Markov chain samples are compared with the experimental reflected signals in Figure 10 for a particular incident angle that was used for the inference. The Fourier transform is also used to compare the spectral density of calculated and experimental signals at the same two angles. Signals are normalized using the maximum value of the experimental signal.

It should be noted that without the extra prior knowledge on the critical angle, the inference outcome is less conclusive, as the ratio $\Lambda^{\prime} / \Lambda$ is clearly not a well identified parameter. As was noted for the identification of material M1, the ratio $\Lambda^{\prime} / \Lambda$ tends to lose sensitivity to the likelihood function as its value increases (asymmetric sensitivity function): once a certain value has been reached, it is virtually impossible to distinguish the likelihood, which makes the inverse problem challenging to solve. Regularizing the inference through prior information is one of the strengths of such a statistical approach, which would have otherwise failed here.

\subsection{M3 identification: low resistivity foam}

The waves reflected from material M3 were measured at 4 different angles of incidence $\left(0^{\circ}, 17^{\circ}\right.$, $\left.23^{\circ}, 35^{\circ}\right)$. Figure 11 shows the pdfs (diagonal elements) and joint-pdfs (extra-diagonal elements) inferred on the material M3 properties. These results are summarized in Table 5 where the MAP and standard deviation of each parameter are reported. Results obtained using classical identification techniques on the same material are also recalled for reference. For highly porous materials such as the one considered here, it is common to have ratios of $\Lambda^{\prime} / \Lambda$ between 2 and 3 . To take this prior 


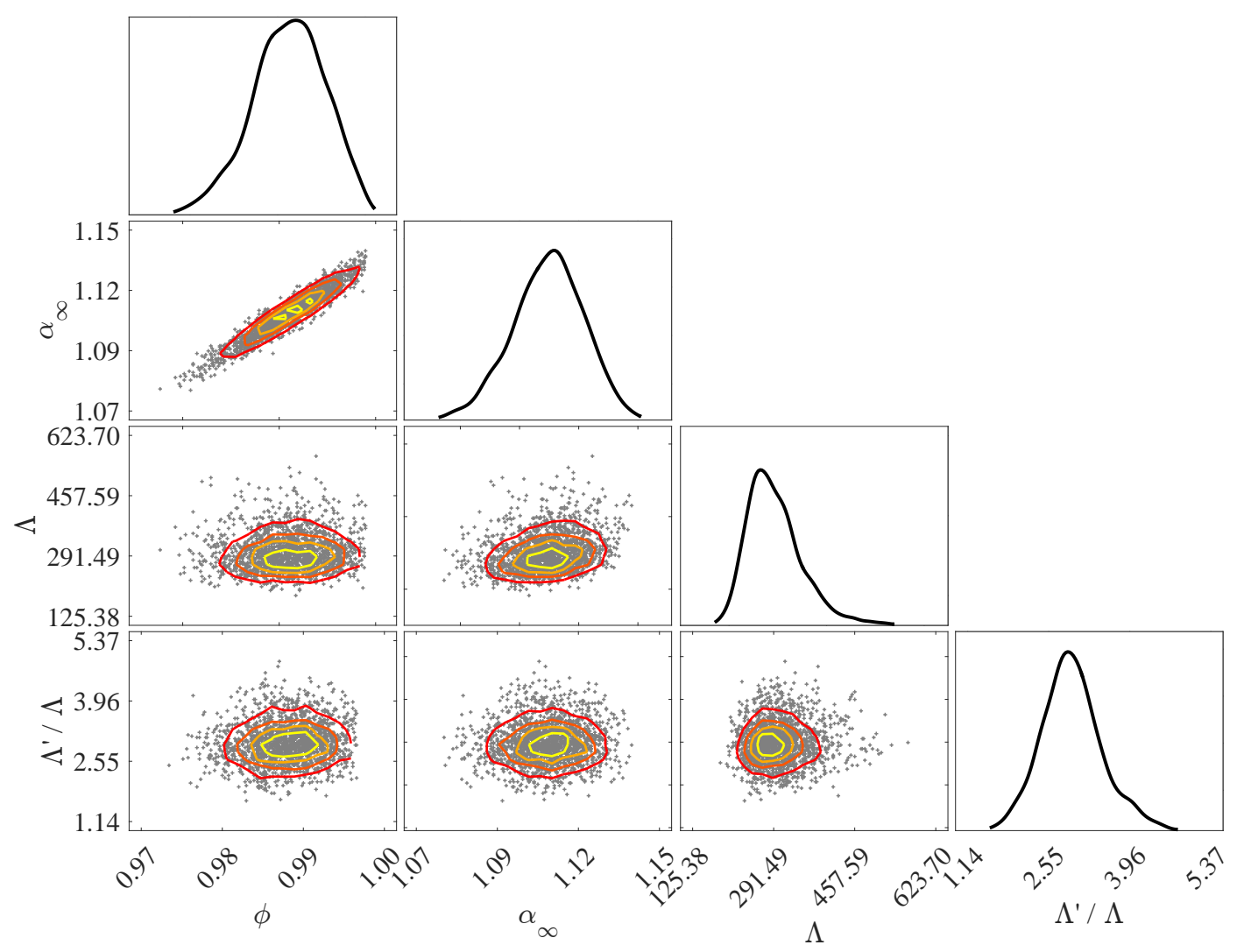

Figure 11: (Color online) M3 inference pdfs (the graphs on the diagonal) and joint-pdfs (the offdiagonal scatter plots)

knowledge into account, the prior density function was updated as

$$
\pi(\boldsymbol{m}) \times \pi_{\Lambda^{\prime} / \Lambda}\left(\Lambda^{\prime} / \Lambda\right) \leftarrow \pi(\boldsymbol{m})
$$

where $\pi_{\Lambda^{\prime} / \Lambda} \sim \mathcal{N}(2.5,1)$. The standard deviation was taken as 1.0 to explain our uncertainty in the guidelines for the prescription in $\Lambda^{\prime} / \Lambda$, and to avoid shifting too strongly the parameter towards the prior: it is estimated that few foam materials such has the one considered here have a ratio of characteristic lengths higher than 4 . The influence of a priori information on the posterior has not been studied further here. The results in Table 4 indicate a posterior probability close to the prior mean, with a standard deviation about half as large. This translates the fact that information from the observed quantities contained identifiable knowledge on $\Lambda^{\prime} / \Lambda$ for us to extract. Despite this prior, the standard deviation of $\Lambda$ remains large, compared with the other inferences performed in this study. Values for the porosity and tortuosity, however, remain well defined and close to the expected values for this type of foam. The observed discrepancies between values can be attributed to the method reaching its limits for this kind of low resistivity material, where the reflected wave amplitude is much smaller than the incident wave amplitude

\begin{tabular}{|c|c|c|c|c|}
\hline Parameter & $\phi$ & $\alpha_{\infty}$ & $\Lambda(\mu \mathrm{m})$ & $\Lambda^{\prime} / \Lambda$ \\
\hline \hline Reference values & 0.97 & 1.06 & 263 & 1.63 \\
\hline Inverted MAP value & 0.99 & 1.11 & 283 & 2.92 \\
\hline Standard deviation & 0.004 & 0.011 & 54.2 & 0.47 \\
\hline
\end{tabular}

Table 5: M3 inference summary. Reference results are obtained with methods developed in [39, 40, 41] 

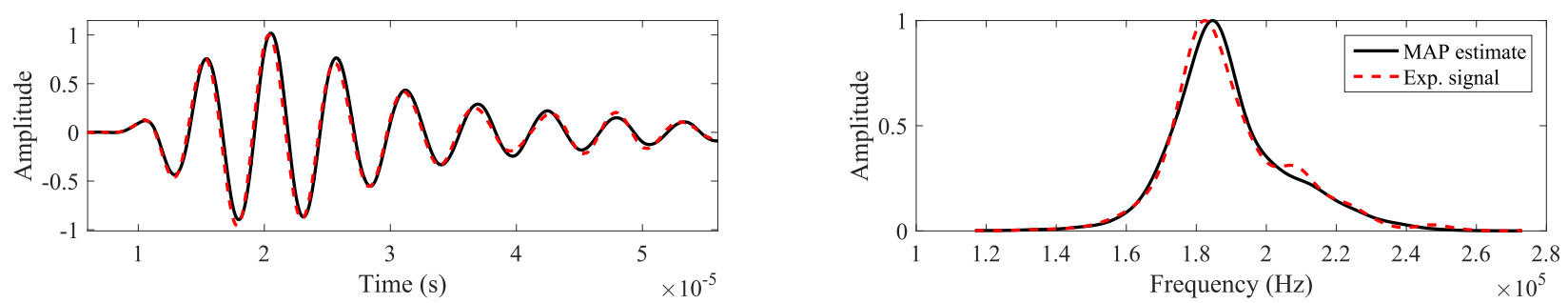

Figure 12: (Color online) Normalized experimental signals : - _ and normalized MAP estimate: — at $\theta_{i}=17^{\circ}$. Left: normalized time signals. Right: normalized frequency spectrum.

The signals calculated using the Markov chain samples are compared with the experimental reflected signals in Figure 12 for a particular incident angle that is used for the inference. The Fourier transform is also used to compare the spectral density of calculated and experimental signals. Signals are normalized using the maximum value of the experimental signal.

In future studies, higher values of incident angles will be used for the identification of low resistivity materials such as this one, as this appears to be the observable region where the dispersive part of the reflection is the greatest.

\subsection{Discussion}

One of the key strengths of Bayesian inference is updating one's belief on a certain hypothesis or parameter through the observation of new data via a robust and coherent approach. In the present work, only a modest use of this strength was tested: the user would certainly benefit from including previous experiments on given materials in terms of informative priors, which would further regularize the inverse problem at hand. While reflected waves were the primary concern of the present study, one is prone to wonder why the transmitted waves were not measured and used as well, in order to gain more information through this observational quantity. This question is especially valid for material M3, a low resistivity foam that is more often tested in transmission measurement mode, since its reflection coefficient is low. Again, the goal set in the present study was purely to introduce a new measuring experiment (the first interface reflected wave at oblique incidence), and a coherent way to aggregate different observations on a given material through first level Bayesian inference. It is of course strongly advised to make use of transmission results whenever readily available. Here, the only additional measurement that was used is the critical angle for which the scattering operator cancels out, for material M2.

\section{Conclusion}

This article proposes a method for the statistical Bayesian inference of homogeneous porous media with a rigid frame, using reflected ultrasonic waves from the first interface in the time domain and the Johnson-Allard model. The reflection operator of the material at the first interface has been worked out in the asymptotic regime in the high frequency limit, for obliquely incident waves. This result has been further analyzed to yield an interesting relationship between a particular angle of incidence, directly relating the tortuosity to the characteristic length ratio of the material. This new knowledge has been used as prior information to improve the regularization of the inverse problem. Furthermore, a detailed analysis of the diffusive kernel of the reflection operator has given new insights into the identification strategy: for highly resistive materials, observing the reflected pressures at low oblique incidence angles is the best strategy, since this is where the diffusive kernel is the most influential; for low resistivity materials, by contrast, it appeared that the diffusive kernel's influence is truly significant mainly for high values of incidence angles, where the full reflection coefficient is greater than for low incidence angles.

In the context of first level Bayesian inference, the information contained in the experimental 
observation and the user knowledge obtained independently of the experiments has been combined to express the posterior probability density of the parameters of interest: the porosity, tortuosity and characteristic lengths. This method offers the possibility to coherently take into consideration prior knowledge of the material properties and to allow uncertainties in the experiment. It is a robust way to take into account new experiments such as the one presented in this study, and combine the extracted knowledge with previous experiments.

Three different materials have been tested and their properties inferred: one granular resistive material of low porosity, one resistive foam of low pore size and medium porosity, and a classical sound absorbing, low resistivity and high porosity foam. It has been shown that using the knowledge of the reflected waves by the first interface only, in the high frequency limit, the extraction of information on the target parameters is possible, even in the case of the low resistivity material where the reflection coefficient is low.

\section{Acknowledgment}

The first author would like to thank the Occitanie region for its financial support during his $\mathrm{PhD}$. C. Depollier is supported by Russian Science Foundation grant number 14-49-00079. The authors appreciate the critical reviews and useful comments made by the anonymous reviewers.

\section{References}

1. J. A. Vrugt, C. J. F. ter Braak, M. P. Clark, J. M. Hyman, B. A. Robinson, Treatment of input uncertainty in hydrologic modeling: Doing hydrology backward with markov chain monte carlo simulation, Water Resour. Res. 44 (12), w00B09. doi:10.1029/2007WR006720.

2. T. Haire, C. Langton, Biot theory: a review of its application to ultrasound propagation through cancellous bone, Bone 24 (4) (1999) 291 - 295. doi:10.1016/S8756-3282(99)00011-3.

3. J. Allard, N. Atalla, Propagation of Sound in Porous Media: Modelling Sound Absorbing Materials 2e, John Wiley \& Sons, 2009. doi:10.1002/9780470747339.

4. M. A. Biot, Theory of propagation of elastic waves in a fluid-saturated porous solid. i. low-frequency range, J. Acoust. Soc. Am. 28 (2) (1956) 168-178. doi:10.1121/1.1908239.

5. M. A. Biot, Theory of propagation of elastic waves in a fluid-saturated porous solid. ii. higher frequency range, J. Acoust. Soc. Am. 28 (2) (1956) 179-191. doi:10.1121/1.1908241.

6. M. A. Biot, Mechanics of deformation and acoustic propagation in porous media, J. Appl. Acoust. 33 (4) (1962) 1482-1498. doi:10.1063/1.1728759.

7. M. A. Biot, Generalized theory of acoustic propagation in porous dissipative media, J. Acoust. Soc. Am. 34 (9A) (1962) 1254-1264. doi:10.1121/1.1918315.

8. D. L. Johnson, J. Koplik, R. Dashen, Theory of dynamic permeability and tortuosity in fluid-saturated porous media, J. Fluid Mech. 176 (1987) 379-402. doi:10.1017/S0022112087000727.

9. Y. Champoux, J.-F. Allard, Dynamic tortuosity and bulk modulus in air-saturated porous media, J. Appl. Acoust. 70 (4) (1991) 1975-1979. doi:10.1063/1.349482.

10. T. G. Zieliński, Normalized inverse characterization of sound absorbing rigid porous media, J. Acoust. Soc. Am. 137 (6) (2015) 3232-3243. doi:10.1121/1.4919806.

11. Y. Atalla, R. Panneton, Inverse acoustical characterization of open cell porous media using impedance tube measurements, Canadian Acoustics 33 (1) (2005) 11-24.

12. P. Cobo, F. Simón, A comparison of impedance models for the inverse estimation of the non-acoustical parameters of granular absorbers, J. Appl. Acoust. 104 (2016) 119-126. doi:10.1016/j.apacoust. 2015.11 .006 
13. T. Hentati, L. Bouazizi, M. Taktak, H. Trabelsi, M. Haddar, Multi-levels inverse identification of physical parameters of porous materials, J. Appl. Acoust.doi:10.1016/j.apacoust.2015.09.013.

14. J. Vanhuyse, E. Deckers, S. Jonckheere, B. Pluymers, W. Desmet, Global optimisation methods for poroelastic material characterisation using a clamped sample in a kundt tube setup, Mechanical Systems and Signal Processing 68-69 (2016) 462 - 478. doi:10.1016/j.ymssp.2015.06.027.

15. Z. E. A. Fellah, S. Berger, W. Lauriks, C. Depollier, C. Aristegui, J.-Y. Chapelon, Measuring the porosity and the tortuosity of porous materials via reflected waves at oblique incidence, J. Acoust. Soc. Am. 113 (5) (2003) 2424-2433. doi:10.1121/1.1567275.

16. Z. E. A. Fellah, S. Berger, W. Lauriks, C. Depollier, P. Trompette, J. Chapelon, Ultrasonic measurement of the porosity and tortuosity of air-saturated random packings of beads, J. Appl. Acoust. 93 (11) (2003) 9352-9359. doi:10.1063/1.1572191.

17. Z. E. A. Fellah, F. G. Mitri, M. Fellah, E. Ogam, C. Depollier, Ultrasonic characterization of porous absorbing materials: Inverse problem, J. Sound. Vib. 302 (4) (2007) 746-759. doi:10.1016/j.jsv. 2006.12.007.

18. J.-P. Groby, E. Ogam, L. De Ryck, N. Sebaa, W. Lauriks, Analytical method for the ultrasonic characterization of homogeneous rigid porous materials from transmitted and reflected coefficients, J. Acoust. Soc. Am. 127 (2) (2010) 764-772. doi:10.1121/1.3283043.

19. Z. E. A. Fellah, M. Sadouki, M. Fellah, F. Mitri, E. Ogam, C. Dépollier, Simultaneous determination of porosity, tortuosity, viscous and thermal characteristic lengths of rigid porous materials, J. App. Phys. 114 (20) (2013) 204902. doi:10.1063/1.4833546.

20. M. Niskanen, J.-P. Groby, A. Duclos, O. Dazel, J. Le Roux, N. Poulain, T. Huttunen, T. Lähivaara, Deterministic and statistical characterization of rigid frame porous materials from impedance tube measurements, J. Acoust. Soc. Am. 142 (4) (2017) 2407-2418. doi:10.1121/1.5008742.

21. J.-D. Chazot, E. Zhang, J. Antoni, Acoustical and mechanical characterization of poroelastic materials using a bayesian approach, J. Acoust. Soc. Am. 131 (6) (2012) 4584-4595. doi:10.1121/1.3699236.

22. C. Zwikker, C. W. Kosten, Sound absorbing materials, Elsevier, 1949.

23. Z. Fellah, C. Depollier, Transient acoustic wave propagation in rigid porous media: A time-domain approach, The Journal of the Acoustical Society of America 107 (2) (2000) 683-688.

24. S. G. Samko, A. A. Kilbas, O. I. Marichev, Fractional integrals and derivatives: theory and applications 44.

25. F. Monteghetti, D. Matignon, E. Piot, L. Pascal, Design of broadband time-domain impedance boundary conditions using the oscillatory-diffusive representation of acoustical models, J. Acoust. Soc. Am. 140 (3) (2016) 1663-1674. doi:10.1121/1.4962277.

26. B. Gurevich, M. Schoenberg, Interface conditions for biot's equations of poroelasticity, The Journal of the Acoustical Society of America 105 (5) (1999) 2585-2589.

27. Z. E. A. Fellah, M. Fellah, W. Lauriks, C. Depollier, Direct and inverse scattering of transient acoustic waves by a slab of rigid porous material, J. Acoust. Soc. Am. 113 (1) (2003) 61-72. doi:10.1121/1. 1528592

28. Z. E. A. Fellah, F. Mitri, C. Depollier, S. Berger, W. Lauriks, J. Chapelon, Characterization of porous materials with a rigid frame via reflected waves, J. Appl. Acoust. 94 (12) (2003) 7914-7922. doi:10.1063/1.1629386.

29. R. C. Smith, Uncertainty quantification: theory, implementation, and applications, Vol. 12, Siam, 2013.

30. A. Tarantola, Inverse problem theory and methods for model parameter estimation, SIAM, 2005. doi:10.1137/1.9780898717921.bm

31. W. R. Gilks, S. Richardson, D. Spiegelhalter, Markov chain Monte Carlo in practice, CRC press, 1995. 
32. N. Metropolis, A. W. Rosenbluth, M. N. Rosenbluth, A. H. Teller, E. Teller, Equation of state calculations by fast computing machines, The journal of chemical physics 21 (6) (1953) 1087-1092. doi:10.1063/1.1699114.

33. W. K. Hastings, Monte carlo sampling methods using markov chains and their applications, Biometrika 57 (1) (1970) 97-109.

34. R. Storn, K. Price, Differential evolution-a simple and efficient heuristic for global optimization over continuous spaces, Journal of global optimization 11 (4) (1997) 341-359. doi:10.1023/A: 1008202821328

35. C. J. Ter Braak, Genetic algorithms and markov chain monte carlo: Differential evolution markov chain makes bayesian computing easy (revised), Tech. rep., Wageningen UR, Biometris (2005).

36. C. J. Ter Braak, A markov chain monte carlo version of the genetic algorithm differential evolution: easy bayesian computing for real parameter spaces, Statistics and Computing 16 (3) (2006) 239-249. doi:10.1007/s11222-006-8769-1.

37. E. Laloy, J. A. Vrugt, High-dimensional posterior exploration of hydrologic models using multipletry dream(zs) and high-performance computing, Water Resour. Res. 48 (1) (2012) n/a-n/a, w01526. doi:10.1029/2011WR010608,

38. A. Gelman, D. B. Rubin, Inference from iterative simulation using multiple sequences, Statistical science 7 (4) (1992) 457-472. URL http://www . jstor.org/stable/2246093

39. Y. Champoux, M. R. Stinson, G. A. Daigle, Air-based system for the measurement of porosity, J. Acoust. Soc. Am. 89 (2) (1991) 910-916. doi:10.1121/1.1894653.

40. N. Brown, M. Melon, V. Montembault, B. Castagnède, W. Lauriks, P. Leclaire, Evaluation of the viscous characteristic length of air-saturated porous materials from the ultrasonic dispersion curve, Comptes rendus de l'Académie des sciences. Série IIb, Mécanique 322 (2) (1996) 122-127.

41. P. Leclaire, L. Kelders, W. Lauriks, M. Melon, N. Brown, B. Castagnede, Determination of the viscous and thermal characteristic lengths of plastic foams by ultrasonic measurements in helium and air, J. App. Phys. 80 (4) (1996) 2009-2012. doi:10.1063/1.363817.

42. N. Xiang, C. Fackler, Objective bayesian analysis in acoustics, Acoustics Today 11 (2) (2015) 54-61.

43. S. R. Pride, F. D. Morgan, A. F. Gangi, Drag forces of porous-medium acoustics, Phys. Rev. B 47 (9) (1993) 4964-4978. doi:10.1103/PhysRevB.47.4964. 